\title{
Dependence on parameters for nonlinear equations - abstract principles and applications
}

\author{
Michał Bełdziński ${ }^{1}$, Marek Galewski ${ }^{1}$, and Igor Kossowski ${ }^{1}$ \\ ${ }^{1}$ Lodz University of Technology Faculty of Technical Physics Information Technology and \\ Applied Mathematics
}

June 22, 2021

\begin{abstract}
We provide parameter dependent version of the Browder-Minty Theorem in case when the solution is unique utilizing different types of monotonicity and compactness assumptions related to condition (S)2. Potential equations and the convergence of their Euler action functionals is also investigated. Applications towards the dependence on parameters for both potential and non-potenial nonlinear Dirichlet boundary problems are given.
\end{abstract}

\section{Hosted file}

Bel_Gal_Kos-Dependence_on_Parameter.pdf available at https://authorea.com/users/421004/ articles/527185-dependence-on-parameters-for-nonlinear-equations-abstract-principlesand-applications 